2021

Non-destructive Correlative 3D

Characterization of Nuclear Graphite:

From the Micro-scale to the Nano-scale

\title{
Kelly, S
}

http://hdl.handle.net/10026.1/17732

10.1007/978-3-030-65261-6_50

TMS 2021 150TH ANNUAL MEETING \& EXHIBITION SUPPLEMENTAL PROCEEDINGS

Springer International Publishing

All content in PEARL is protected by copyright law. Author manuscripts are made available in accordance with publisher policies. Please cite only the published version using the details provided on the item record or document. In the absence of an open licence (e.g. Creative Commons), permissions for further reuse of content should be sought from the publisher or author. 


\title{
Non-destructive Correlative 3D \\ Characterization of Nuclear Graphite: \\ From the Micro-scale to the Nano-scale
}

\author{
Stephen Kelly, Robin White, William Harris, Tobias Volkenandt, \\ Benjamin Tordoff, Giuliano Laudone, Katie Jones, and Ben Veater
}

\begin{abstract}
Graphite is a key material in the design and operation of a wide range of nuclear reactors because of its attractive combination of thermal, mechanical, and neutron interaction properties. In all its applications, the microstructural evolution of nuclear graphite under operating conditions will strongly influence reactor lifetime and performance. However, measuring the $3 \mathrm{D}$ microstructural characteristics of nuclear graphite has traditionally faced many challenges. X-ray tomographic techniques face limitations in achievable resolution on bulk (mm-sized) specimens while serial sectioning techniques like FIB-SEM struggle to achieve adequate milling rates for tomographic imaging over representative volumes. To address these shortcomings, we present here a multiscale, targeted, correlative microstructural characterization workflow for nuclear graphite employing micro-scale and nano-scale x-ray microscopy with a connected laser milling step in between the two modalities. We present details of the microstructure, including porosity analysis, spanning orders of magnitude in feature size for nuclear graphite samples including IG-110.
\end{abstract}

Keywords Graphite $\cdot$ X-ray tomography $\cdot$ Porosity $\cdot$ Multiscale

\section{Introduction}

The advent of the femtosecond (fs) laser and its application to material ablation tasks has proven to be a game changer for materials research. With their extremely rapid milling rates (orders of magnitude faster than traditional ion beam approaches) and minimal heat affected zone (HAZ), the fs-laser has brought about a renaissance in

\author{
S. Kelly $(\bowtie) \cdot R$. White $\cdot$ W. Harris \\ Carl Zeiss X-ray Microscopy, Pleasanton, CA, USA \\ e-mail: steve.kelly@zeiss.com \\ T. Volkenandt $\cdot$ B. Tordoff \\ Carl Zeiss RMS, Oberkochen, Germany \\ G. Laudone $\cdot$ K. Jones $\cdot$ B. Veater \\ University of Plymouth, Plymouth, UK
}


advanced materials characterization capabilities. Recently, a fs-laser mill has been integrated into a commercial focused ion beam and scanning electron microscope (FIB-SEM) instrument, enabling numerous new capabilities, including access to deeply buried structures as well as production of extremely large trenches, cross sections, pillars, and TEM H-bars, all while preserving microstructure and avoiding or reducing FIB polishing. In addition to workflows utilizing the FIB-SEM instrument, this capability enables workflows that span between multiple instruments and the instrument can act as a rapid, targeted sample preparation station to enable a wide range of such workflows. For example, workflows moving from 3D imaging with $\mathrm{X}$-ray microscopy (XRM) into high resolution 2D or 3D imaging with the FIB-SEM can now target deeply buried (more than 50-100 $\mu \mathrm{m}$ below the surface) structures for high resolution analysis via FIB-SEM. Also, workflows moving from micro-scale 3D XRM (samples generally a few millimetres in size) into nano-scale 3D XRM (samples generally $\sim 100 \mu \mathrm{m}$ in size) can leverage the instrument to rapidly prepare nano-scale XRM samples in a targeted manner. This latter workflow is the subject of this work.

As a testbed for this workflow development and implementation, we use nuclear grade graphite. Nuclear grade graphites are high purity forms of graphite that have been engineered for several critical uses in a range of nuclear reactors. Graphite has a unique combination of properties that make it attractive for uses in nuclear energy generation, including robust thermal and mechanical properties, and attractive neutron interaction cross sections. Graphite finds uses as, for example, neutron moderators and structural components in fission reactors such as the high-temperature gas-cooled design, and neutron reflectors in developing fusion reactor designs.

In all these applications, the specific 3D microstructural properties of the graphite used can significantly affect the performance of the reactor and the ageing and degradation properties of the graphite itself. For example, the internal porosity of the graphite material can significantly affect several important material parameters relevant to reactor operation. The amount of porosity can affect the mechanical properties and thermal transport properties of the graphite. The structure and shape of the porosity can affect the way in which the graphite corrodes and oxidizes in the reactor, leading to a compromise in its integrity. As such, understanding the $3 \mathrm{D}$ microstructure of nuclear grade graphite is essential to understanding the performance and ageing characteristics of this critical material and providing insight for ways to engineer new forms of graphite through modelling and to improve performance in the future. Porosity in nuclear grade graphite and its evolution with exposure to operating conditions, including that studied here, has been studied extensively both computationally and experimentally $[1,2]$.

At the same time, however, nuclear grade graphite poses several challenges to traditional materials imaging techniques. First, the pore structure is highly multiscale in nature, spanning over several orders of magnitude in pore size. Second, the low sputtering yields and extreme hardness of the material make large scale material removal, such as that leveraged in this workflow, impractical with ion beam based approaches. 
The workflow and results outlined in this work overcome these challenges in two ways. The challenge of multi-scale pore structure imaging is addressed by leveraging both micro-scale and nano-scale XRM to provide the needed resolutions for comprehensive 3D imaging. Meanwhile, the material removal and sample preparation challenges are addressed by leveraging the capabilities of a connected, correlative fs-laser mill integrated into a FIB-SEM instrument to prepare a portion of the sample imaged with micro-scale XRM for subsequent imaging with nano-scale XRM.

Together, this 3D-to-3D x-ray imaging workflow spans over 10x in observable length scales and sample volumes imaged and enables a holistic multi-scale analysis not possible with one modality alone. While the analysis presented here reveals microstructural characteristics of nuclear graphite, the workflow itself is general enough to be applied to many other systems across materials science.

\section{Methods}

\section{Materials}

Commercial nuclear grade graphite of type IG-110 (Toyo Tanso Co., Ltd., Japan) was used for these experiments presented here. IG-110 is an isotropic graphite derived from petroleum coke and is manufactured by cold isotropic molding (isomolding) producing needle-shaped filler particles of $\approx 20 \mu \mathrm{m}$. IG-110 grade graphite is used as a neutron moderator and reflector in components for high temperature gas-cooled reactors [3] and is currently in use in the High Temperature engineering Test Reactor (HTTR) in Japan [4] and the HTR-10 in China [5].

\section{Sample Preparation}

To facilitate the sample preparation, roughly $1 \times 1 \times 1 \mathrm{~mm}$ sized pieces were manually cut from the bulk material using an IsoMet low speed precision cutter (Buehler, Lake Bluff, IL). Using 5-min epoxy, single pieces were glued on separate standard $5 \mathrm{~mm}$ SEM stubs for further sample preparation using Zeiss Crossbeam 350 laser (LaserFIB) (Carl Zeiss Microscopy GmbH, Jena, Germany). The LaserFIB features an integrated femtosecond laser for targeted laser preparation in a dedicated chamber attached to the airlock of the main FIB-SEM. After co-registering the laser and FIB-SEM sample chamber coordinate systems and selecting a region of interest by SEM imaging, the sample was transferred to the laser chamber and a two-step laser preparation process was carried out. Firstly, laser scanning pattern was setup using CAD software so that a layer of about $300 \mu \mathrm{m}$ thickness was removed top-down over the whole sample area of about $1 \mathrm{~mm}^{2}$, leaving out a circular area of about $200 \mu \mathrm{m}$ in diameter. This resulted in a remaining rough pillar of $300 \mu \mathrm{m}$ in height protruding 
from the sample surface. This pillar was laser polished towards suitable dimensions in the second step. Here, a ring-shaped ("donut") laser pattern with a chosen inner diameter to give a final diameter of $50 \mu \mathrm{m}$ at the top was positioned around the pillar. While the pattern of the first step was rastered line-wise with a rotation of the line direction by $19^{\circ}$ after each complete layer to achieve homogeneous material removal, the second step used a spiral raster from outside to inside to polish the shape of the pillar. 250 raster layers at a scan speed of $200 \mathrm{~mm} / \mathrm{s}$ were used in the rough milling step and the polishing step consisted of 300 raster layers at $50 \mathrm{~mm} / \mathrm{s}$. The laser power was set to $8 \mathrm{~W}$ and the pulse frequency to $50 \mathrm{kHz}$ in both cases.

\section{Micro-scale X-ray Imaging}

X-ray tomographic imaging was performed using Zeiss Xradia 620 Versa X-ray Microscope (XRM) (Carl Zeiss X-ray Microscopy Inc., Pleasanton, CA). The XRM employs a tungsten target anode with potential accelerating voltage range of $30 \mathrm{kV}$ to $160 \mathrm{kV}$. Here, an accelerating voltage of $60 \mathrm{kV}$ was used. During the tomography, 2401 projection images were acquired over an angle range of-94 to +94 degrees utilizing the $4 \mathrm{x}$ optical magnification objective with an exposure of $10 \mathrm{~s}$ per image. This resulted in a pixel size $0.7 \mu \mathrm{m}$ and field-of-view of $1.4 \mathrm{~mm}$. Reconstruction was performed using commercial software package XMReconstructor (Carl Zeiss Xray Microscopy Inc., Pleasanton, CA) utilizing a cone-beam filtered back-projection algorithm.

\section{Nano-scale X-ray Imaging}

High resolution X-ray tomographic imaging was performed using Zeiss Xradia 810 Ultra X-ray Microscope (XRM) (Carl Zeiss X-ray Microscopy Inc., Pleasanton, CA). The unique architecture of the XRM uses a sequence of X-ray optics to achieve spatial resolution of $50 \mathrm{~nm}$ or below allowing for high resolution imaging and high contrast sensitivity to nondestructively visualize the internal morphology. The XRM employs a Chromium target anode with pseudo-monochromatic x-rays at $5.4 \mathrm{keV}$, as well, Zernike phase contrast imaging was performed. Images were acquired with $64 \mathrm{~nm}$ pixels and a $64 \mu \mathrm{m}$ field of view. For the tomography, 901 projection images collected at $60 \mathrm{~s}$ each were acquired. Reconstruction was performed using commercial software package XMReconstructor (Carl Zeiss X-ray Microscopy Inc., Pleasanton, CA) utilizing a parallel-beam filtered back-projection algorithm (Figs. 1, 2 and 3). 

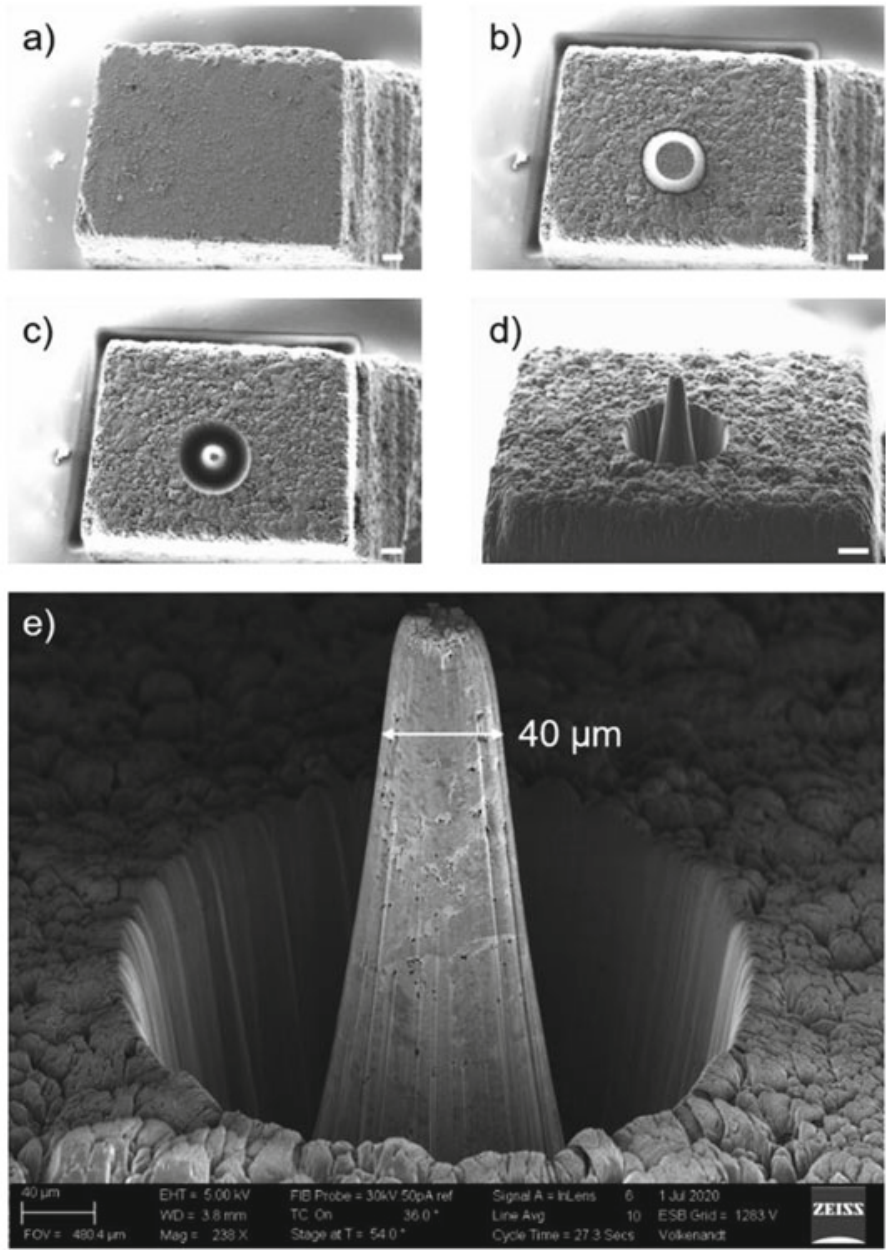

Fig. 1 SEM micrographs of the pillar preparation process. a the initial surface of the millimeter sized chunk, $\mathbf{b}$ after milling the $\sim 200 \mu \mathrm{m}$ diameter pillar into the surface, $\mathbf{c}$ after laser polishing to reduce the diameter to a suitable size for nano-scale XRM, $\mathbf{d}$ shows a side view of the pillar with the entire chunk in the background, $\mathbf{e}$ shows the completed pillar in detail, including the clean sidewalls resulting from the laser ablation. Scale bars in $\mathbf{a}-\mathbf{d}$ are $100 \mu \mathrm{m}$

\section{Image Processing/Analysis/Visualization}

All visualization and image processing was performed using Dragonfly Pro software (ORS, Montreal, Canada). Versa and Ultra datasets were manually aligned. Large grains observed in the Versa data were isolated using a combination of grayscale thresholding, morphological operations, and a distance map transform to approximate the grain boundaries. Initially, pores were segmented by thresholding greyscale 


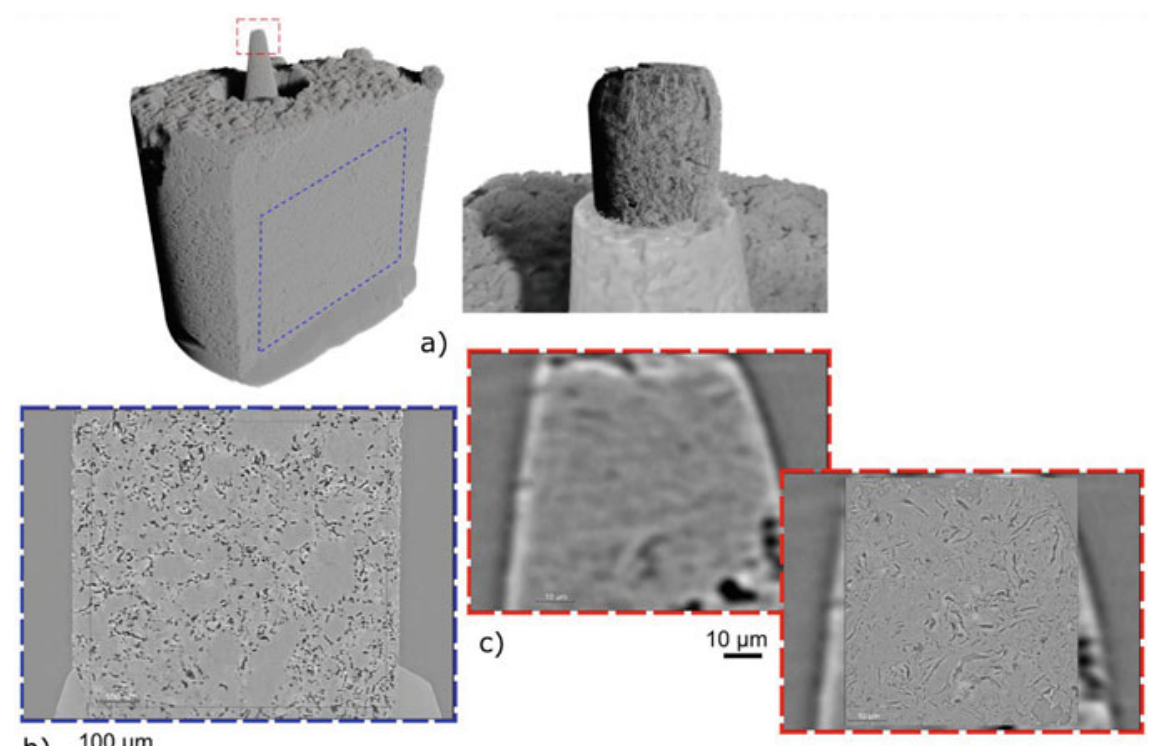

b) $100 \mu \mathrm{m}$

Fig. 2 Micro-scale and nano-scale XRM results, a shows a 3D rendering of the micro-scale (left) and nano-scale (right) XRM data, $\mathbf{b}$ shows a representative slice through the interior of the microscale XRM data, c shows the pillar region prepared via laser milling in both the micro-scale (left) and nano-scale (right) XRM data. (Color figure online)

values. Following this, an opening operation was used to remove small pores. Closing and dilation steps were performed to identify regions of high porosity density which define boundaries for the targeted grain regions. A pixel wise distance map was then calculated. This distance transform was segmented to form seeds of visual centers of the grain regions. A watershed transform was subsequently performed to grow the grain boundaries over the defined solid space. Porosity was quantified using grayscale thresholding followed by pore segmentation using the OpenPNM library implemented in Dragonfly Pro [6] for both Versa and Ultra datasets.

\section{Results}

As described in Sect. "Sample Preparation", the millimeter-sized chunk of graphite was milled to form a pillar at the top of the sample suitable for measurement with nano-scale XRM. The steps taken to produce the pillar are shown in Fig. 1a-d, while the resulting pillar can be seen in Fig. 1e. The top portion of the pillar is $<50 \mu \mathrm{m}$ in diameter, ideal for imaging with nano-scale XRM. The graphite grain structure and porosity can be seen on the surface of the pillar, indicating the high quality of the laser-prepared surface. Total laser ablation time to produce the pillar was $750 \mathrm{~s}$. 


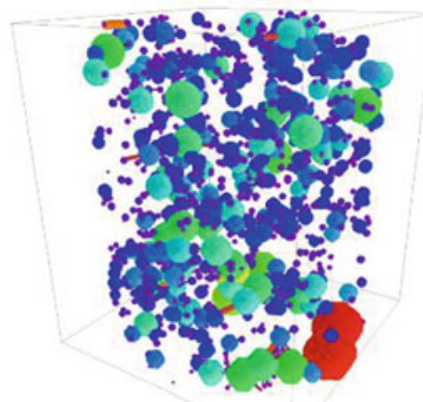

a)

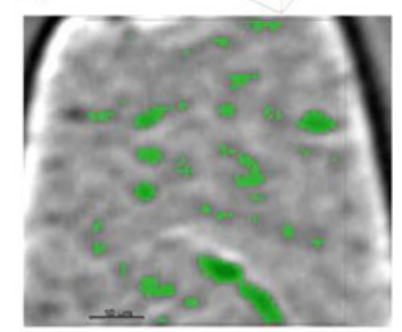

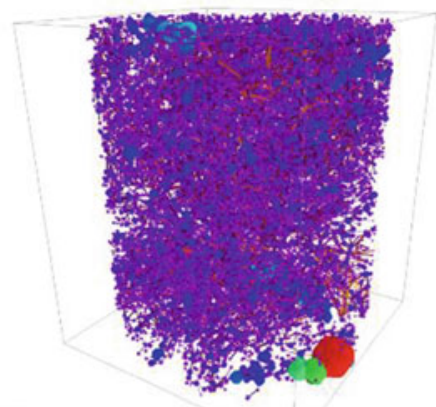

b)

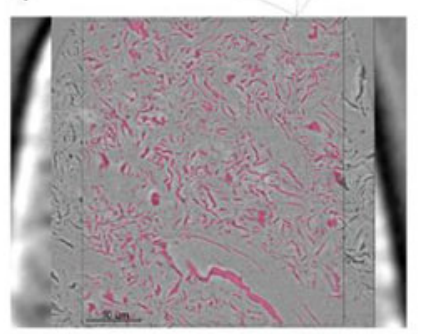

Fig. 3 Comparison of porosity segmented and measured in the same region for a micro-scale XRM and $\mathbf{b}$ nano-scale XRM. The lower images show an example slice through the data revealing the segmentation results in detail. The upper images show a connectivity diagram for the pore networks segmented in each volume where colored balls represent the pores and the sticks represent connectivity between the pores. (Color figure online)

Figure 2 shows the results of both the micro-scale and nano-scale XRM measurements on the IG-110 graphite sample. While the micro-scale XRM data captured information across the entire sample volume, the nano-scale XRM data is localized within the prepared pillar. Figure 2a shows a 3D rendering of the entire sample volume, with the side artificially clipped to reveal the interior of the millimeter sized base. The dotted blue and red insets show the regions highlighted in b) and c), respectively. Figure $2 b$ shows the internal microstructure of the IG-110 graphite in detail. Distinct regions are visible in the image that represent high density graphite grains separated by a more porous region where the binder material has bound them together in the formation process. Very little porosity is visible in the more dense regions in the micro-scale XRM data. In Fig. $2 \mathrm{c}$ the resolution improvements between the micro-scale and nano-scale XRM are shown in detail.

Strikingly, the area shows a high degree of internal porosity in the nano-scale data which is not visible in the micro-scale XRM data. As a further example of the additional information revealed with the nano-scale XRM, Fig. 3a-b show pore segmentation for the same volume across both micro-scale and nano-scale XRM, respectively. The lower images of Fig. 3 clearly show the additional resolved porosity obtained in the nano-scale XRM data. Table 1 lists porosity values for this volume as measured in both the micro-scale and nano-scale XRM data. The micro-scale 


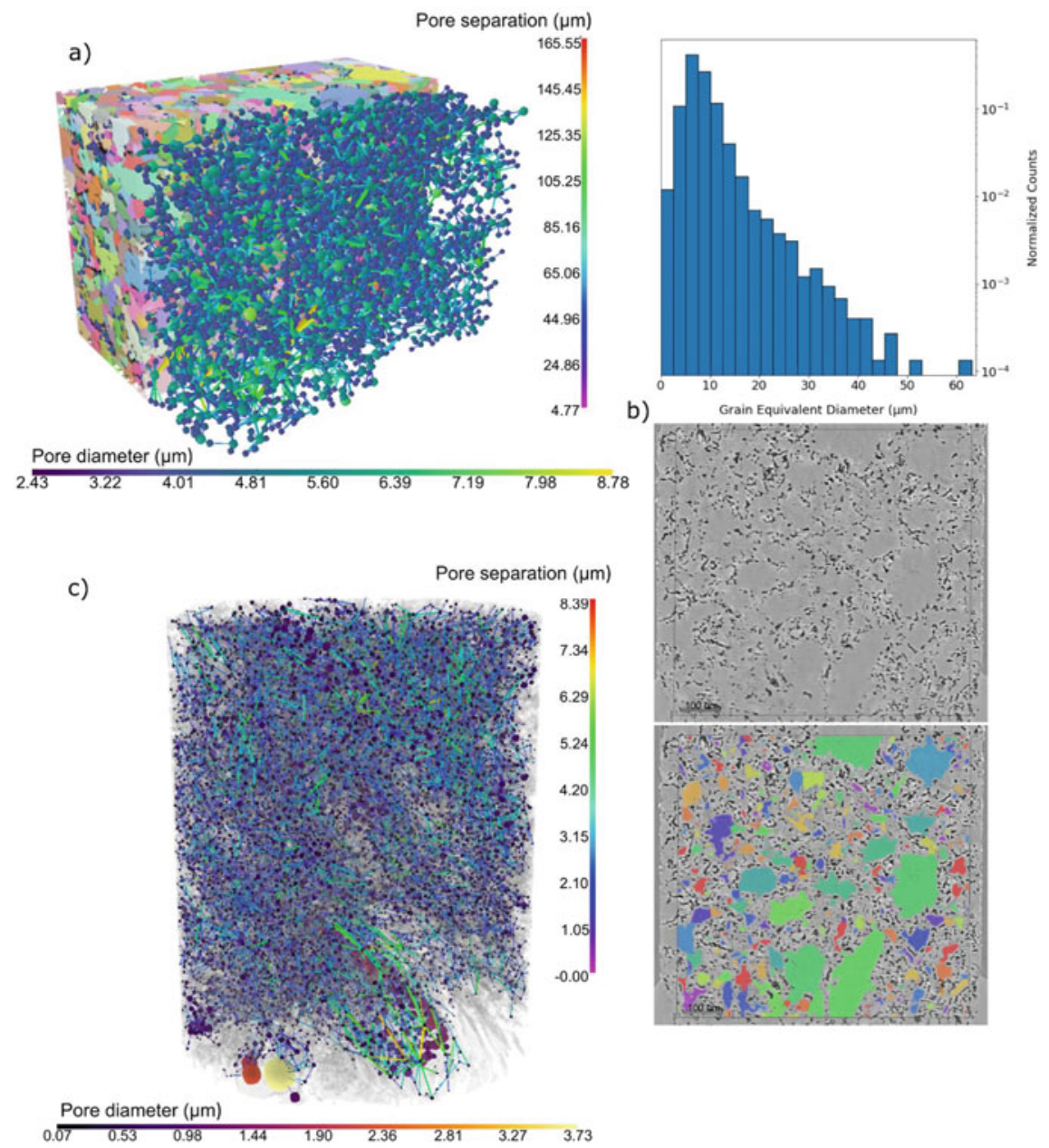

Fig. 4 Multiscale data analysis on the measured pore network. Part a shows the segmented dense grain regions (colored blocks in the rear) along with the 3D pore network model of the porosity in the micro-scale XRM data while the plot to the right shows the segmented grain size distribution. Part $\mathbf{b}$ shows the result of the grain segmentation overlaid in color (bottom) onto a single slice taken from the micro-scale XRM data (top). Part $\mathbf{c}$ shows the pore network model from the nano-scale XRM data. (Color figure online)

Table 1 Porosity metrics for the data described here

\begin{tabular}{l|l|l|l|l}
\hline Dataset & $\begin{array}{l}\text { Analysis sub } \\
\text { volume }(\mu \mathrm{m})\end{array}$ & Porosity $(\%)$ & $\begin{array}{l}\text { Grain Volume } \\
\text { Fraction }(\%)\end{array}$ & $\begin{array}{l}\text { Total Interpolated } \\
\text { Porosity }(\%)\end{array}$ \\
\hline Micro-scale XRM & $\begin{array}{l}740 \times 730 \times \\
1055\end{array}$ & 12.8 & 49.5 & 19.7 \\
\hline Micro-scale XRM & $24 \times 24 \times 56$ & 6.5 & & \\
\hline Nano-scale XRM & $24 \times 24 \times 56$ & 15.5 & & \\
\hline
\end{tabular}


XRM data would indicate that this dense region contains only $6.5 \%$ porosity, while the (more accurate) nano-scale XRM data reveals the porosity to be over twice that amount at $15.5 \%$. Furthermore, the pore network models of the two segmented data sets in the upper images of Fig. 3 reveal that not only are the larger pores from the micro-scale XRM data actually composed of several smaller pores, but that the micro-scale XRM data does not capture the connectivity of the pore network (absence of sticks connecting the spheres) while that character is well captured in the nano-scale XRM data. The connectivity and pore network character for the nanoscale XRM measurement is shown in more detail in Fig. 4c where the ball color represents the pore size and the stick color represents the pore separation distance. The connectivity of the pore network is an essential characteristic for understanding degradation properties in nuclear grade graphite as it dictates how gases can move through the structure and potentially react with the material.

Importantly, the volume analyzed by nano-scale XRM is from the interior of one of the dense grains visible from the micro-scale XRM images. As such, the metrics derived from this region can be used to enhance the metrics across the entire volume of the micro-scale XRM data to gain a much more complete picture of the overall material microstructure. To do this, the dense grain regions were first segmented from the porous regions in the micro-scale XRM data. This segmentation can be seen in the 2D images shown in Fig. $4 \mathrm{~b}$ where the colored patches in the lower image represent the identified grains within the material. These grains are represented as the colored blocks in the background of the 3D rendering of Fig. 4a, with the grain size distribution plotted on the right side of Fig. 4a. The grain regions represent $49.5 \%$ of the total analyzed volume, while the total porosity measured in the micro-scale XRM data is found to be $12.8 \%$, as tabulated in Table 1 . Assuming the dense grain regions contain similar levels of porosity, we can take the $15.5 \%$ porosity metric from the nano-scale XRM data and use that to correct the measured $12.8 \%$ porosity from the micro-scale XRM data. For the grain regions, we assume the porosity is $15.5 \%$ (as measured from the nano-scale XRM data). For the porous regions, we use the porosity value measured from the micro-scale XRM data and assume this captures most of the porosity in this region. Then we sum the pore volume for the entire volume and rescale to the total analyzed volume to report a corrected porosity.

Table 1 summarizes the holistic, multiscale porosity analysis undertaken here. The total interpolated porosity measured with this approach is $19.7 \%$, which matches well with the tabulated literature porosity value for IG-110 of $22.7 \%$ [7, 8]. The discrepancy between the two measurements could be due to segmentation variations, the particular regions analyzed, or finer scale porosity not captured by the nano-scale XRM data.

Aside from simply measuring the porosity value in this material, this multi-scale analysis approach delivers a comprehensive microstructural understanding of the pore network structure in nuclear graphite. This information is critical in developing a theoretical understanding of this critical material in its complex service environment. Additionally, as the sample is still intact following analysis, this approach can be used to track microstructure evolution with exposure to elevated temperatures, neutron irradiation, or corrosive environments. Future work will focus on refining 
this workflow to deliver a more accurate microstructural representation, exploring other nuclear graphite forms, and extending the application of this method to other systems across materials science.

\section{Conclusions}

In conclusion, we present here a targeted, multiscale, correlative 3D imaging workflow spanning between micro-scale and nano-scale XRM and linked by the rapid sample preparation capabilities afforded by a fs-laser mill integrated onto a FIBSEM instrument. The workflow has been used to analyze the multiscale porosity network present in the nuclear grade graphite IG-110. The measured porosity value of $19.7 \%$ from this method closely matches the reported literature value of $22.7 \%$ that was obtained from other methods, but yields a more complete picture of the porosity structure than can be obtained by non-imaging methods. The resulting multi-scale porosity representation makes useful input for modelling approaches that simulate the response of nuclear graphite to different operational conditions.

\section{References}

1. Jones KL, Laudone GM, Matthews GP (2018) A multi-technique experimental and modelling study of the porous structure of IG-110 and IG-430 nuclear graphite. Carbon 128(1):1-11

2. Snead L, Contescu C, Byun T, Porter W (2016) Thermophysical property and pore structure evolution in stressed and non-stressed neutron irradiated IG-110 nuclear graphite. J Nucl Mater 476(4):102-109

3. Xu Y, Zuo K (2002) Overview of the $10 \mathrm{MW}$ high temperature gas coolded reactor-test module project. Nucl Eng Des 218(1-3):13-23

4. Shiozawa S, Fujikawa S, Iyoku T, Kunitomi K, Tachibana Y (2004) Overview of HTTR design features. Nucl Eng Des 233(1-3):11-21

5. Wu Z, Lin D, Zhong D (2002) The design features of the HTR-10. Nucl Eng Des 218(1-3):25-32

6. Gostick J et al. (2016) OpenPNM: A pore network modeling package. Comput Sci Eng 18(4):6074

7. Oku T, Eto M, Ishiyama S (1990) Irradiation creep properties and strength of a fine-grained isotropic graphite. J Nucl Mater 172(1):77-84

8. Burchell T (1996) A microstructurally based fracture model for polygranular graphites. Carbon 34(3):297-316 